\title{
UM ESTUDO SOBRE A INFLUÊNCIA DE PROPRIEDADES FÍSICAS DA ESCÓRIA E DE PARÂMETROS OPERACIONAIS SOBRE O PROCESSO DE SLAG SPLASHING*
}

\author{
Paula Maria Gomes Cunha Leão ${ }^{1}$ \\ Eliana Ferreira Rodrigues ${ }^{2}$ \\ Carlos Antonio da Silva ${ }^{3}$ \\ Itavahn Alves da Silva ${ }^{3}$
}

\section{Resumo}

Um modelo matemático tridimensional, via software Fluent 14.0, de modo a descrever o fluxo, em regime transiente e isotérmico dos fluidos escória e nitrogênio, foi utilizado para avaliar o comportamento de slag splashing em um convertedor a oxigênio com o propósito de reparar as regiões desgastadas do revestimento de trabalho deste reator. Para um dado padrão de sopro foi investigou-se o efeito de parâmetros tais como temperatura, densidade, viscosidade e tensão interfacial. Os resultados são comparados com dados de projeção disponíveis na literatura.

Palavras-chave: Convertedor a oxigênio; Slag splashing; Modelagem matemática.

\section{A STUDY ON THE INFLUENCE OF PHYSICAL PROPERTIES OF SLAG AND OF OPERATIONAL PARAMETERS ON THE SPLASHING SLAG PROCESS \\ Abstract}

A mathematical model using Fluent 14.0 was implemented in order to describe the flow of Nitrogen and slag under transient and isothermal conditions related to slag splashing. The purpose was to study the influence of parameters affecting the repair of the working refractory lining. For a given blow pattern the influence of parameters such as temperature, density, viscosity and interfacial tension was assessed. The results are compared with projection data available in the literature.

Keywords: Basic oxygen furnace; Slag splashing; Mathematical modelling.

Aluna de graduação, Curso de Engenharia Metalúrgica e de Materiais, Escola de Minas, Universidade Federal de Ouro Preto, Minas Gerais, Brasil.

2 Engenheira Mecânica, DSc., Professora, Escola de Minas, Universidade Federal de Ouro Preto, Escola de Minas, Minas Gerais, Brasil.

3 Engenheiro Metalúrgico, Ph.D., Professor, DEMET, Escola de Minas, Universidade Federal de Ouro Preto, Escola de Minas, Minas Gerais, Brasil. 


\section{INTRODUÇÃO}

As causas de desgaste do revestimento refratário de trabalho de um convertedor a oxigênio são decorrentes da combinação de fenômenos térmicos, químicos e mecânicos. As causas térmicas são atreladas às flutuações de temperatura e choques térmicos; enquanto que as causas de degradação de natureza química decorrem das interações químicas entre o refratário e a escória e atmosfera do convertedor. Já aquelas de origem mecânica são associadas ao impacto de sucata, erosão durante movimentação do reator; condições de sopro de oxigênio e da instalação do revestimento refratário. Por meio da técnica de monitoramento do perfil de desgaste do revestimento refratário por feixe de laser, Figura 1, obtém-se o mapa do perfil de desgaste do revestimento refratário em cada região do convertedor. A escala de cores indica o valor máximo e o mínimo da espessura do revestimento refratário (em $\mathrm{mm}$ ) para cada região do convertedor. Nota-se que as regiões de corrida e de impacto de sucata são as mais susceptíveis à degradação. Com isto, pode-se estabelecer uma estratégia de reparo adequado das regiões desgastadas, prolongando a campanha do reator.
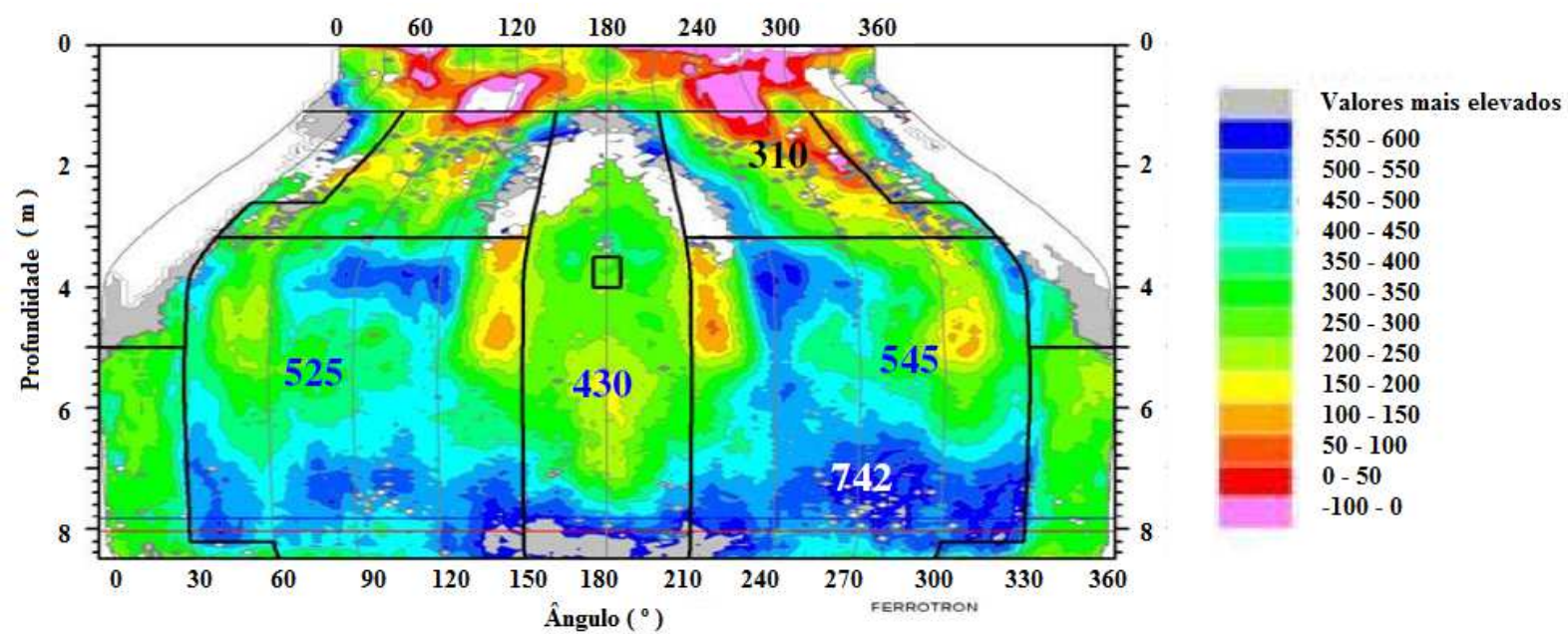

[Fonte: www.frost.com/prod/servlet/cio/116975323, acessado em 27/07/214]

Figura 1 - Valores máximo e mínimo da espessura do revestimento refratário nas cinco regiões do convertedor a oxigênio, via monitoramento por feixe de laser.

As técnicas de manutenção e reparo do revestimento refratário incluem recobrimento ou lavagem com escória; projeção de escória via lança de sopro de nitrogênio (slag splashing) e gunitagem. De acordo com Mills et al. [1]' Strelbisky \& Manning [2], o processo de slag splashing é caracterizado pela projeção de escória quimicamente recondicionada, via um fluxo de nitrogênio, contra a face quente do revestimento de trabalho do convertedor. Muitos fatores afetam o processo de projeção de escória tais como: dimensões do convertedor; velocidade do sopro de Nitrogênio; altura, inclinação e geometria do bocal da lança de nitrogênio; temperatura, composição e volume de escória. A duração desta prática é de 1 a 4 minutos, portanto, mais rápida do que a técnica de recobrimento por escória (slag coating). Atualmente a campanha de um convertedor com revestimento refratário reparado por "slag plashing" alcança 10.000 corridas ou mais. Mais recentemente Sabah \& Brooks [3] estudaram, via modelagem física, a influência dos parâmetros citados sobre a trajetória e distribuição de tamanhos das gotas ejetadas neste processo. Koopmans et al. [4] apontam que existem uma altura e vazão de nitrogênio ótimas para que a taxa de projeção de gotas de escória e a espessura da camada de escória sejam otimizadas nas diversas regiões críticas de 
desgaste do revestimento refratário do convertedor. Alam et al. [5] apontam que, desde que a vazão crítica de gás para ejeção das gotas é independente da altura da lança, o fator contribuinte do aumento da geração de gotas de escória deve ser a mudança da área superficial da cavidade formada pelo sopro do gás, Figura 2. He \& Standish [6] apontam que a geração de gotas por de ser por: respingamento (geração de gotas individuais e discretas) ou por enxame de gotas (produção de várias gotas de vários tamanhos). Por outro lado, Molloy [7] e Kumagai \& Iguchi [8] argumentam que o aumento da velocidade de sopro do gás e/ou a redução da altura da lança de sopro do gás podem provocar a redução da quantidade de gotas ejetadas de escória. Isto pode ser atribuído ao aumento da profundidade da cavidade gerada pelo do jato gasoso devido ao aumento da pressão dinâmica do jato gasoso. Estes eventos provocam o aparecimento de oscilação e vorticidade da escória.

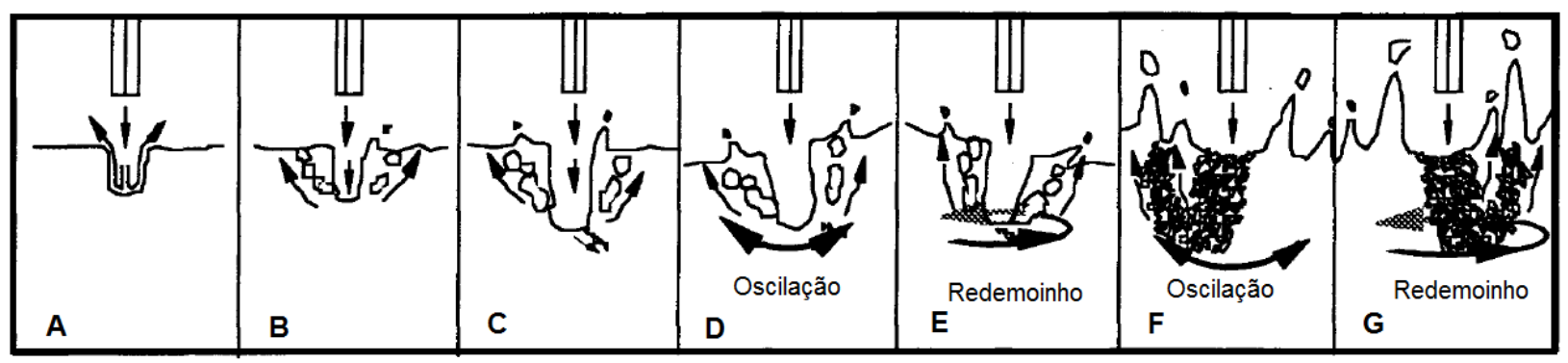

Figura 2 - Características da interação fase líquida e um jato gasoso vertical [8]

\section{METODOLOGIA}

Como base para os cálculos foram utilizadas as equações de Navier-Stokes, o modelo de turbulência $k-\varepsilon$, juntamente com a formulação de fração volumétrica -VOF (para rastreamento da posição da interface entre nitrogênio/escória). Onde se encontra a presença de mais de uma fase de fluido, a formulação VOF é usada para expressar a interações entre as interfaces das fases de gás/escória.

Sendo os fluidos incompressíveis e Newtonianos, o fluxo é descrito por:

$$
\rho\left(\frac{\partial \vec{u}}{\partial t}+\vec{u} \cdot \nabla \vec{u}\right)=-\nabla p+\mu_{e f f} \nabla^{2} \vec{u}+\rho \vec{g}+F,
$$

onde: $\rho=$ densidade do fluido, $\vec{u}=$ vetor velocidade, $t=$ tempo, $g=$ gravidade, $p=$ pressão, $F$ são as outras forças(de contato entre as fases) que atuam no fluido, e $\mu_{e f f}$ $=$ viscosidade efetiva do fluido.

A computação envolvendo o modelo $\boldsymbol{K}-\boldsymbol{\varepsilon}$ envolve resolver as equações:

$$
\begin{gathered}
\rho \frac{\partial K}{\partial t}+\rho v_{j} \frac{\partial K}{\partial x_{j}}=\frac{\partial}{\partial x_{j}}\left(\frac{\mu_{t}}{\sigma_{K}} \frac{\partial K}{\partial x_{j}}\right)+\mu_{t} \frac{\partial v_{j}}{\partial x_{i}}\left(\frac{\partial v_{i}}{\partial x_{j}}+\frac{\partial v_{j}}{\partial x_{i}}\right)-\rho \varepsilon \\
\rho \frac{\partial \epsilon}{\partial t}+\rho v_{j} \frac{\partial \varepsilon}{\partial x_{j}}=\frac{\partial}{\partial x_{j}}\left(\frac{\mu_{t}}{\sigma_{\varepsilon}} \frac{\partial_{\varepsilon}}{\partial x_{j}}\right)+C_{1} \mu_{t} \frac{\varepsilon}{K} \frac{\partial v_{j}}{\partial x_{i}}\left(\frac{\partial v_{i}}{\partial x_{j}}+\frac{\partial v_{i}}{\partial x_{i}}\right)-C_{2} \frac{\varepsilon}{K} \rho \varepsilon
\end{gathered}
$$

Onde: $\quad \boldsymbol{K}=$ a energia cinética de turbulência, $\boldsymbol{\varepsilon}=$ a taxa de dissipação da energia cinética de turbulência. A viscosidade efetiva é determinada a partir de:

$$
\mu_{e f f=} \mu_{0}+\mu_{t}
$$

onde, $\boldsymbol{\mu}_{\mathbf{0}}$ representa a viscosidade molecular e a viscosidade turbulenta é dada por:

$$
\mu_{t}=\frac{\rho C_{\mu} K^{2}}{\varepsilon}
$$


A localização da interface escória líquida/nitrogênio é determinada pela formulação fração volumétrica VOF, e a equação da continuidade para um par de fases é representada por:

$$
\frac{\partial}{\partial t}\left(r_{\alpha} \rho_{\alpha}\right)+\nabla \cdot\left(\mathrm{r}_{\alpha} \rho_{\alpha} \mathrm{U}_{\alpha}\right)=0
$$

onde: $\boldsymbol{r}=$ a fração em volume e $\boldsymbol{\rho}=$ a densidade.

Considera-se que a soma total da fração de volume seja unitária, ou seja:

$$
\sum_{\alpha} r_{\alpha}=1
$$

Para reduzir o esforço computacional, um $1 / 4$ de um conversor isotérmico tridimensional foi considerado, Figura 3 . As equações foram resolvidas por meio do software Ansys FLUENT. O algoritmo segregado (baseado na pressão) foi utilizado juntamente com o esquema implícito de linearização. Para que se obtivesse uma solução mais precisa foi empregado o esquema Second-order Upwind na discretização das equações governantes. Foi utilizado, ainda, o algoritmo PRESTO para a interpolação dos valores da pressão nas faces das células usando a equação da quantidade de movimento e o algoritmo PISO para a obtenção do acoplamento pressão-velocidade. Utilizou-se uma malha tetraédrica composta de 627.535 células. O tempo máximo de processamento para todas as simulações foi de 2 segundos com um timestep de $1 \times 10^{-5}$ segundos.

\section{RESULTADOS}

Os resultados obtidos mostram um comportamento errático das projeções de gotas de escória. Isto pode ser associado ao comportamento transiente e instável da zona de impacto do jato gasoso sobre a escória. Este tipo de comportamento foi também citado por Liu et al. [9], Barrón et al [10,11]. A Figura 3 mostra o efeito da densidade da escória sobre o comportamento de projeção da mesma. Nota-se maior densidade da escória incorre em menor taxa de projeção. Liu et al.[9] ressaltam que menor viscosidade da escória possibilita o aumento da profundidade de penetração do jato gasoso e maior altura de projeção das gotas de escória. Contudo, escórias mais fluidas tendem a escorrer pela parede do convertedor e afinar a camada de recobrimento. A qualidade da projeção (altura, número de gotas projetadas e espessura da camada de recobrimento) é fortemente afetada pela viscosidade da escória recondicionada. Altos valores de viscosidade incorrem em menor taxa de projeção, o que poderia requerer maiores temperaturas e vazões de nitrogênio. Portanto, a boa eficiência de reparo das regiões desgastadas do revestimento refratário de trabalho do convertedor exige a adequação da composição e temperatura da escória para se inicie o processo de slag splashing. E sob um ponto de vista operacional, o recobrimento das paredes laterais superiores do convertedor (regiões dos munhões e cone superior) pode ser melhorado pelo aumento da velocidade de sopro de nitrogênio e pela diminuição da densidade da escória. 

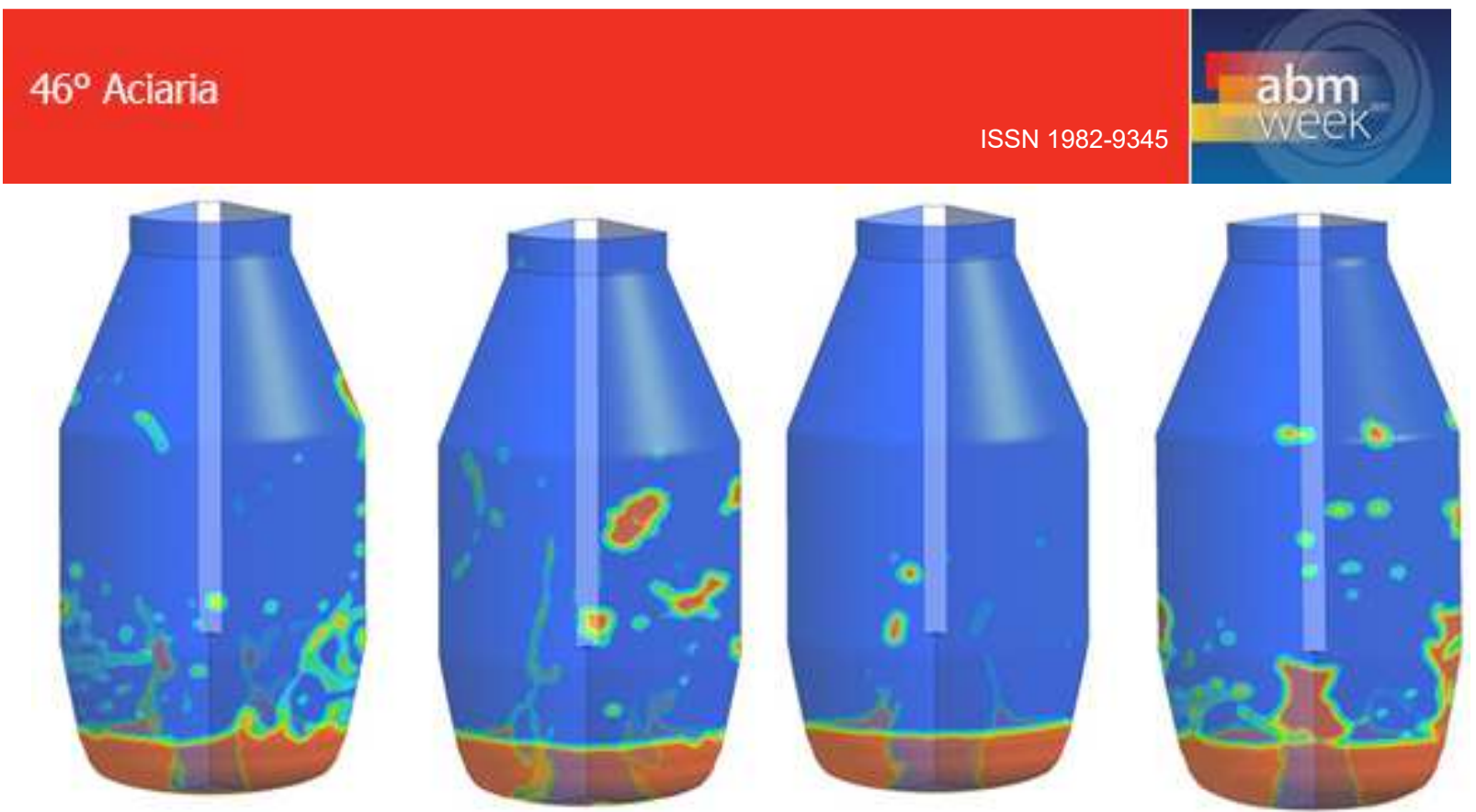

Figura 1 - Projeção de escória contra parede do convertedor viscosidade 0,058N.s.m $\mathrm{m}^{-2}$ e densidade de escória de a) $3490 \mathrm{~kg} / \mathrm{m}^{3}$ e b) $2500 \mathrm{~kg} / \mathrm{m}^{3}$; viscosidade $1 \mathrm{~N} . \mathrm{s} . \mathrm{m}^{-2}$ e massa específica de (c) $3490 \mathrm{~kg} / \mathrm{m}^{3}$ e (d) $2500 \mathrm{~kg} / \mathrm{m}^{3}$.

Para as condições operacionais investigadas, os resultados obtidos mostram que a projeção de escória é espacialmente irregular, Figura 4 e que o aumento da viscosidade da escória decresce a taxa de projeção.
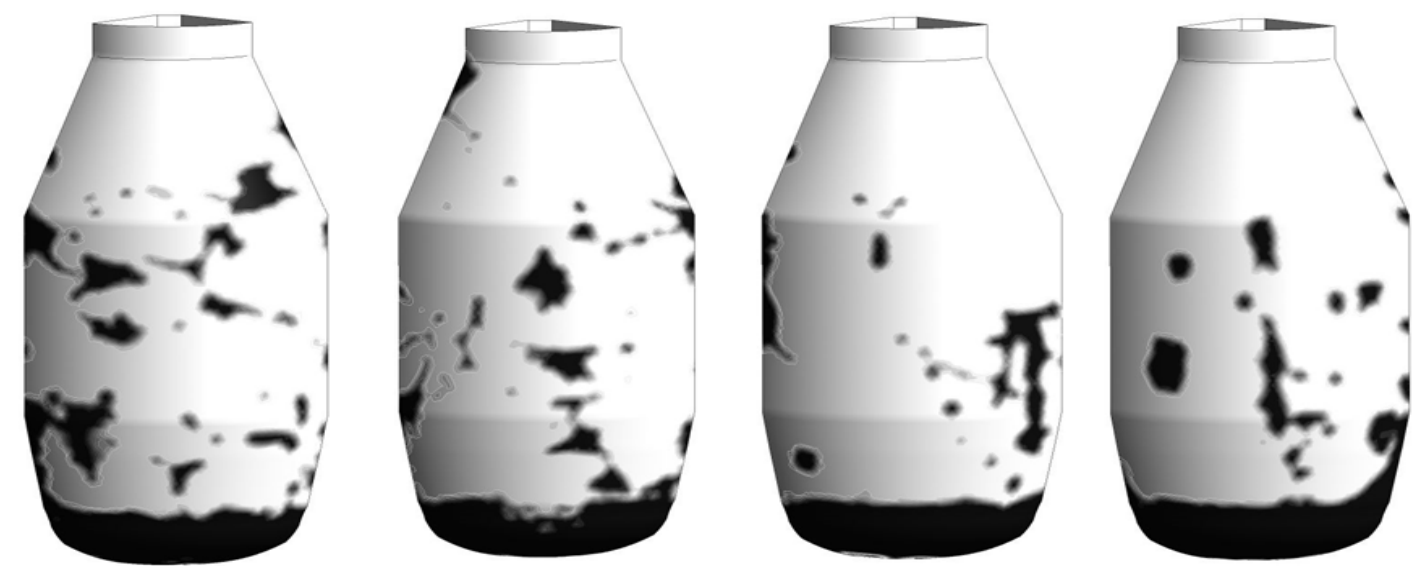

Figura 2- Fração de recobrimento por escória na parede do convertedor no tempo de 2 segundos para viscosidades cinemáticas de a) $23 \times 10^{-6} \mathrm{~m}^{2} . \mathrm{s}^{-1}$; b) $40 \times 10^{-6} \mathrm{~m}^{2} . \mathrm{s}^{-1}$; c) $200 \times 10^{-6} \mathrm{~m}^{2} \cdot \mathrm{s}^{-1}$ e d) $400 \times 10^{-6} \mathrm{~m}^{2} . \mathrm{s}^{-1}$.

Liu et al.[9] realizaram estudos envolvendo modelagem física do fenômeno de slag splashing. Seus resultados mostram a influência do padrão de sopro (altura lança escória, taxa de sopro pelo fundo, taxa de sopro pelo topo), geometria do bocal da lança, do grau de preenchimento do reator pela escória residual, e das propriedades físicas da escória sobre a taxa de projeção de escória.

O aumento da viscosidade decresce a quantidade de escória projetada (e aderida) para as regiões superiores do refratário de trabalho do convertedor. Este comportamento é previsto, seguindo o trabalho de Liu et al.[9], na Figura 5. 


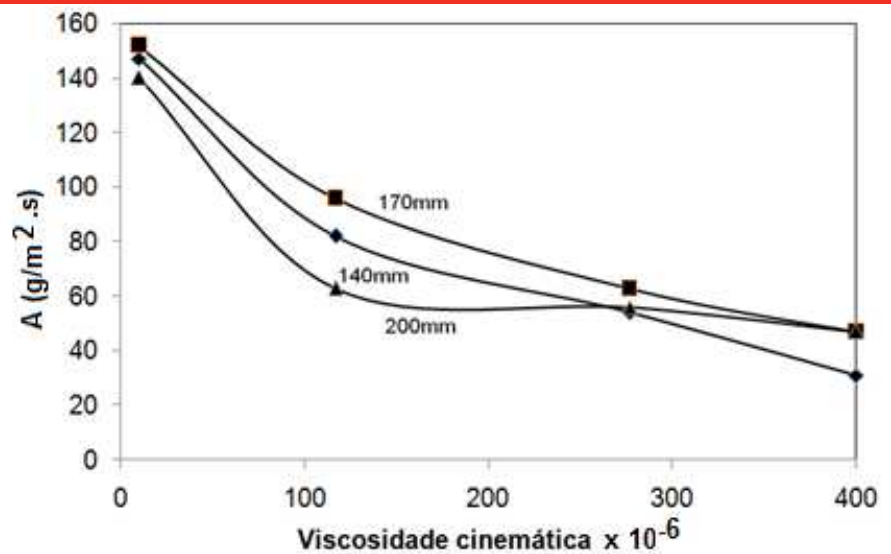

Figura 3 - Efeito da viscosidade cinemática sobre a taxa de projeção de escória, altura da lança de $140 \mathrm{~mm}, 170 \mathrm{~mm}$ e $200 \mathrm{~mm}$, a partir dos resultados obtidos por Liu et al.[9].

A partir da análise dos resultados experimentais, obtidos por Liu et al. [9], obtidos via modelagem física, a taxa de projeção de escória $\left(A\right.$, em $\left.\mathrm{g} / \mathrm{m}^{2} \mathrm{~s}\right)$ em função da altura da lança em relação à camada de escória $(\mathrm{h}$, em $\mathrm{mm})$ e da viscosidade cinemática da escória $\left(v\right.$, em $\left.10^{-6} \mathrm{~m}^{2} / \mathrm{s}\right)$, os autores deste trabalho obtiveram a seguinte correlação:

$$
\begin{gathered}
A=-0.6-0.286 v+1.42 h-0.00371 h^{2}+0.000572 v^{2}-0.000953 v h \\
\left(R^{2}=0,82\right)
\end{gathered}
$$

a qual indica que, para uma dada viscosidade da escória, existe uma altura da lança em que a taxa de projeção de escória é máxima.

A Tabela 1 mostra o fluxo específico de escória como função da altura da ponta da lança e da viscosidade da escória. Nota-se que o fluxo é maior na região inferior do convertedor (linha de escória), comparativamente com a região do munhão e do cone superior. Este resultado condiz com o observado por Liu et al. [9], se for considerada a aplicação do fator de escala para correção do valor da distância banho escória. De acordo com estes autores a região da linha de escória recebe $66,8 \%$ do material projetado; a do munhão recebe $29,2 \%$ e a do cone superior cerca de $3 \%$. Nota-se ainda que o aumento da viscosidade da escória decresce a taxa de projeção na região da linha de escória.

Tabela 1: Fluxo de massa de escoria em planos transversais do convertedor valores em $\mathrm{kg} \cdot \mathrm{m}^{-2} \cdot \mathrm{s}^{-1}$ em $1 / 4$ de área para um tempo de 1,2 s de sopro.

\begin{tabular}{|c|c|c|}
\hline & \multicolumn{2}{|c|}{ Altura da lança em relação à camada de escória } \\
\hline Viscosidade da escória & $1 \mathrm{~m}$ & $2 \mathrm{~m}$ \\
\hline $0,1 \mathrm{~N} . \mathrm{s} \cdot \mathrm{m}^{-2}$ & $0,0621 \mathrm{kgm}^{-2} \mathrm{~s}^{-1}$ & $0,0008 \mathrm{kgm}^{-2} \mathrm{~s}$ \\
\hline $1 \mathrm{~N} . \mathrm{s} \cdot \mathrm{m}^{-2}$ & $0,0552 \mathrm{kgm}^{-2} \mathrm{~s}^{-1}$ & $0,0008 \mathrm{kgm}^{-2} \mathrm{~s}$ \\
\hline
\end{tabular}

Como a área da superfície interna do convertedor investigado é de $126,6 \mathrm{~m}^{2}$, de acordo com a Figura 6, para uma escória com viscosidade cinemática e $23 \times 10^{-6} \mathrm{~m}^{2} / \mathrm{s}$, o tempo necessário para recobrir o revestimento refratário de trabalho deste reator seria de 93s(1,55min.). Liu et al.[9] destacam que a esta duração do processo deve-se ainda acrescentar o período de gestação de $1 / 4$ a $1 / 2$ do tempo efetivo de slag splashing. Este tempo de gestação, que não foi considerado na modelagem matemática, deriva da diminuição de temperatura e de viscosidade, além da emulsificação da escória, durante todo o sopro de nitrogênio. Então, a duração de recobrimento do convertedor considerado seria maior do que de 1,83 - 2,32min. Esta duração está compatível com a prática operacional mencionada por Mills et al.[1], a qual varia de 2 a $4 \mathrm{~min}$. 


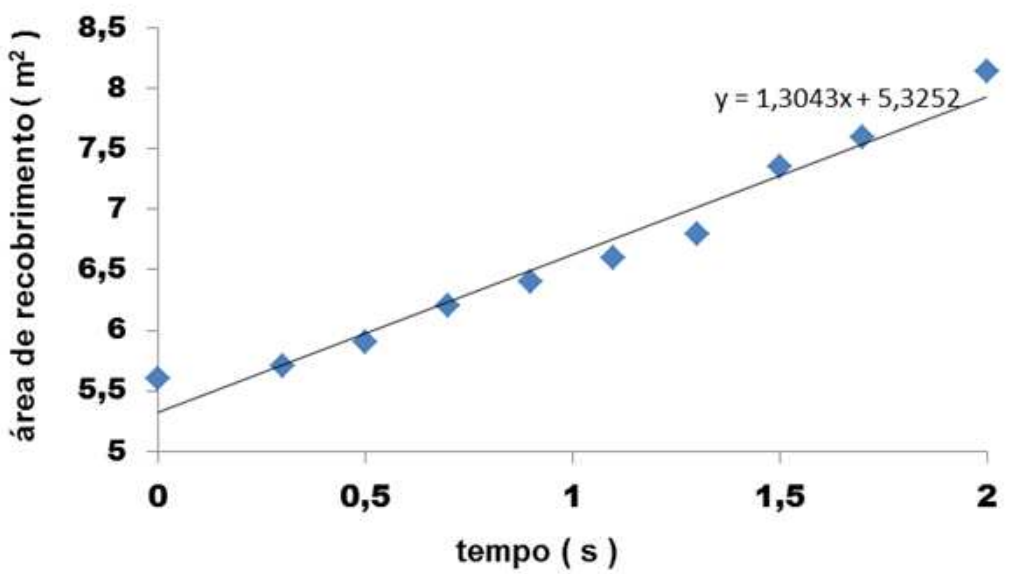

Figura 4 - Área de recobrimento da parede do convertedor em $t=2 s$ iniciais de sopro e viscosidade cinemática da escória igual a $23 \times 10^{-6} \mathrm{~m}^{2} / \mathrm{s}$.

O aumento da viscosidade cinemática decresce a área de recobrimento pela escória, Figura 7. Contudo, o aumento da área recoberta com a diminuição da viscosidade da escória, embora facilite maior altura das gotas projetadas, incorre em afinamento da camada de recobrimento o que é prejudicial ao reparo do revestimento refratário. Este comportamento foi observado por Barrón et al.[10], Liu et al. [9] em decorrência do fato de que a taxa de projeção decresce com o aumento da densidade ou da viscosidade. Por isso, que Liu et al.[9] destaca a necessidade de que a viscosidade da escória de projeção deve ser conveniente ajustada pela adição de ingredientes reguladores desta propriedade física da escória.

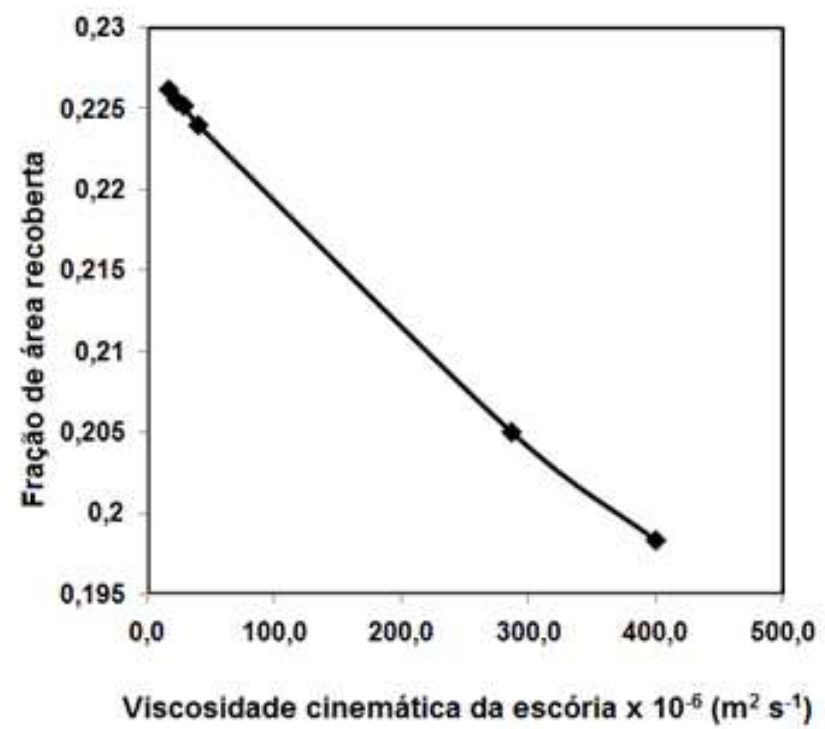

Figura 5 - Efeito da fração de área recoberta do revestimento de trabalho com a viscosidade da escória

Liu et al. [9], a partir de modelagem física, cita que apenas 46,8\% da quantidade de escória é efetivamente empregada no recobrimento do revestimento refratário. Tomando como referência, um convertedor de 340toneladas de aço com 40 toneladas de escória de refino [12], conforme a premissa anterior de Liu et al. [9], cerca de 18,7 toneladas desta escória seriam empregadas no reparo do revestimento interno do convertedor. Mills et al.[1] citam um valor de cerca 14 toneladas de escória projetada. À guisa de simplicidade, admitindo que a espessura do recobrimento seja uniforme e a área do revestimento refratário do convertedor de $126,6 \mathrm{~m}^{2}$, a quantidade de escória 
aderida seria de $147,70 \mathrm{~kg} / \mathrm{m}^{2}$. Contudo, os resultados de modelagem matemática mostraram que a camada de recobrimento apresentava espessura variável.

\section{DISCUSSÃO}

Os resultados da modelagem matemática demonstraram a natureza errática e heterogênea da projeção de gotas de escória e, por consequência, a não uniformidade espacial do recobrimento do revestimento de trabalho do convertedor. Sabah \& Brooks [3] Liu et al. [9], Subagyo et al.[13], Koria \& Lange [14] apontam que a qualidade de ejeção de gotas de escória (número, tamanho) depende da instabilidade da cavidade formada pelo impacto o jato gasoso com a escória. Mills et al. [1] destacam que a instabilidade da cavidade, sob o ponto de vista prático, pode ser representada pelos adimensionais de Weber (We) e de momento (Mn):

$$
W e=\frac{\rho_{\text {gás }} u_{g}^{2}}{\sqrt{\sigma g \rho_{\text {esc }}}} \quad M n=\frac{\rho_{\text {gás }} A_{\text {orif }} u_{g}^{2}}{\rho_{\text {esc }} g h_{\text {cav }}}
$$

$\rho_{\text {esc }}, \rho_{\text {gás }}, A_{\text {orif }}, u_{g}, g, h_{\text {cav }}=$ massas específicas da escória e do gás; área do orifício de injeção do gás; velocidade do jato gasoso, aceleração da gravidade e profundidade da cavidade gerada pelo do jato de nitrogênio sobre a camada de escória, respectivamente.

Mills et al. [1], Alam et al.[5], Liu et al.[9] destacam que os efeitos negativos da densidade e viscosidade da escória sobre a eficiência de recobrimento por projeção podem ser contrabalanceados pela adequação do padrão de sopro de nitrogênio (altura e geometria do bocal da lança, vazão do gás). Com isto, podem-se proporcionar forças adicionais de momento, melhorando a formação de pequenas gotas de escória densa, favorecendo o recobrimento das regiões superiores do convertedor. Para um aumento da reação entre profundidade da cavidade e a altura da camada de escória $\left(\mathrm{h}_{\text {cav }} / \mathrm{h}_{\text {esc }}\right)$, de acordo com Mills et al.[1], Liu et al.[9], incorrerá em aumento da altura de projeção das gotas de escória. Com a variação da velocidade de sopro do gás pode-se alterar a taxa de geração e a distribuição de tamanho de gotas de escória, em benefício da qualidade do recobrimento. Subagyo et al.[13] destacam que a taxa de projeção de escória pode ser correlacionada como o adimensional de sopro (Blowing Number), NB, definido como:

$$
N B=\frac{\rho_{g} u_{g}^{2}}{2 \sqrt{\sigma g \rho_{\text {esc }}}}
$$

Quando NB > 1 ocorre a formação de gotas. De acordo com Sabah \& Brooks [3], para as condições industriais de slag splashing, NB varia entre 4,11 e 6

Finalmente, como o perfil térmico no convertedor é transiente e heterogêneo, Figura 8; e como os adimensionais que caracterizam a geração e a projeção de gotas de escória (We, Mn e NB) dependem de propriedades físicas da escória (viscosidade, tensão interfacial e densidade), além da taxa de solidificação da escória de recobrimento do refratário, são afetadas pela temperatura. Por isso, é importante ainda investigar os efeitos do perfil térmico no convertedor sobre as características de instabilidade da zona de impacto do jato de nitrogênio e da qualidade da área recoberta por escória. Com isto, para uma dada condição operacional de sopro de nitrogênio; composição e nível de superaquecimento da escória podem-se investigar os efeitos do perfil de temperatura no convertedor sobre a altura de projeção e a taxa de aderência das gotas de escória. 

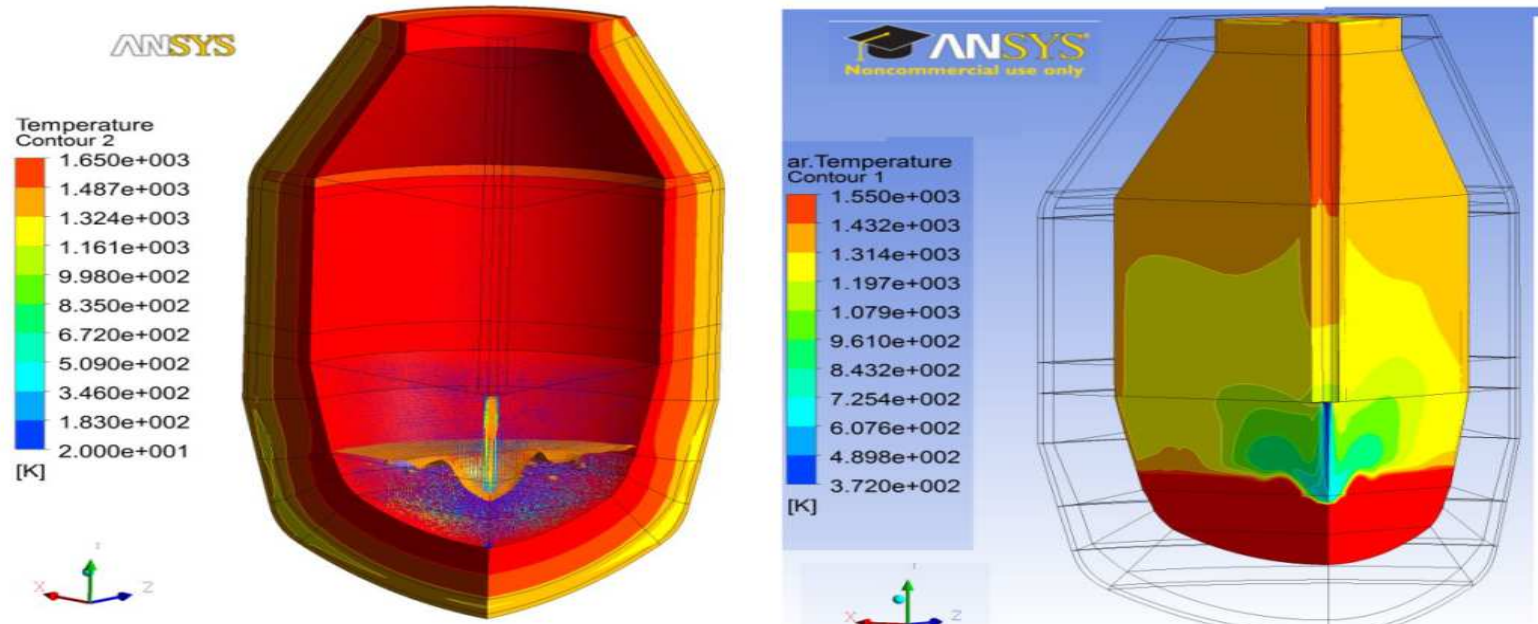

Figura 6 - Distribuição de temperatura no interior do convertedor durante a operação de projeção de escória

\section{CONCLUSÕES}

A eficiência do processo slag splashing requer a adequação da vazão de sopro e posicionamento da lança, da distribuição de temperatura na face quente do revestimento de trabalho; da composição mineralógica da escória do convertedor de modo a balancear as propriedades físicas viscosidade, tensão interfacial e densidade. Os resultados indicam o forte efeito da viscosidade da escória sobre a taxa de projeção. A taxa de projeção de gotas aumenta com a diminuição da viscosidade da escória e com a diminuição da densidade.

\section{Agradecimentos}

Ao programa de Iniciação Científica a UFOP, à Fundação Gorceix, Fapemig, Capes e CNPq.

\section{REFERÊNCIAS}

1 Mills, K.C; Su, Y; Fox, A.B; Li, Z; Thackray, R.P \& Tsai, H. T - A Review of Slag Splashing," ISIJ International, Vol. 45, No. 5, 2005, pp. 619-633.

2 Strelbisky,Mi.J \& Manning, J - Current slag splashing practices in selected, http://www.tallman-bronze.com/articles/article_2/article-slag.htm, postado em 2005, acessado em 01/03/2015

3 Sabah S. And Brooks G. Splashing in Oxygen Steelmaking. ISIJ International, Vol. 54, No.4, 2014, pp. 836-844.

4 Koopmans, P; Mcdonald, C; Drugge, J \& Vatanen, J - Primary Steelmaking: Consistent BOS performance. Technical Steel Research, EUR2081, 2003

5 Alam, M; Naser, J; Brooks, \& Fontana, A - A Computational Fluid Dynamics Model of Shrouded Supersonic Jet Impingement on a Water Surface. ISIJ International, Vol. 52 (2012), No. 6, pp. 1026-1035

$6 \mathrm{He}, \mathrm{Q}$. L \& Standish, N-Model study of droplet generation in the BOF steelmaking. ISIJ International, vol. 30, no. 4, 1990, pp. 305-309.

7 Molloy, N.A - Impinging jet flow in a two-phase system. The basic flow pattern. J. Iron Steel Inst, vol. 208, no. 10, 1970. pp. 943-950.

8 Kumagai, T \& M. Iguchi, M - Instability phenomena at bath surface induced by top lance gas injection," ISIJ International, vol. 41, pp. 52-55, 2001.

9 Liu, C.J; Zhu, Y. X \& Jiang. M. F - Physical modelling of slag splashing in converter, Ironmaking and Steelmaking. vol. 30 No. 1, 2003,p. 36-42 
10 Barron, M.A; Medina, D. Y \& Hilerio, I - CFD Analysis of Influence of Slag Viscosity on the Splashing Process in an Oxygen Steelmaking Converter Modeling and Numerical Simulation of Material Science, 2013, 3, 90-93

11 Barron, M.A; Medina, D.Y; Hilerio, I \& Plascencia, G - Influence of the Slag Density on the Splashing Process in a Steelmaking Converter;. ISRN Metallurgy, 2014, p. 1-7

12 Odenthal, H-J; Falkenreck, U \& Schlüter, J - CFD simulation of multiphase melt flows in Steelmaking converters. European Conference on Computational Fluid Dynamics ECCOMAS CFD 2006. Pp. 1-21

13 Subagyo, Brooks, G.A; Coley, K \& Irons, G - Generation of droplets in slag-metal emulsions through top gas blowing. ISIJ International, vol. 43, no. 7, 2003, pp. 983-989.

14 Koria, S \& Lange, K - A new approach to investigate the drop size distribution in basic oxygen steelmaking. Metallurgical Transactions B, vol. 15, no. 1, pp. 109-116, 1984. 